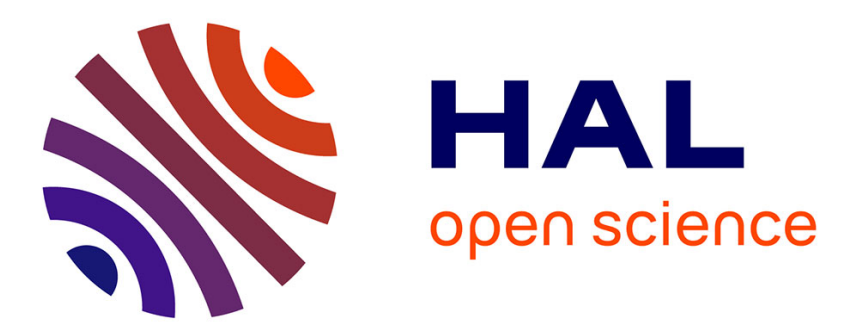

\title{
LES JUGES FRANÇAIS ET LA GESTATION POUR AUTRUI
}

\author{
Martine Gross, Laurence Brunet, Michelle Giroux
}

\section{To cite this version:}

Martine Gross, Laurence Brunet, Michelle Giroux. LES JUGES FRANÇAIS ET LA GESTATION POUR AUTRUI. Perspectives internationales sur la gestation pour autrui. Expériences des personnes concernées et contextes d'action, 2018. hal-01723910

\section{HAL Id: hal-01723910 https://hal.science/hal-01723910}

Submitted on 5 Mar 2018

HAL is a multi-disciplinary open access archive for the deposit and dissemination of scientific research documents, whether they are published or not. The documents may come from teaching and research institutions in France or abroad, or from public or private research centers.
L'archive ouverte pluridisciplinaire HAL, est destinée au dépôt et à la diffusion de documents scientifiques de niveau recherche, publiés ou non, émanant des établissements d'enseignement et de recherche français ou étrangers, des laboratoires publics ou privés. 


\title{
LES JUGES FRANÇAIS ET LA GESTATION POUR AUTRUI
}

\author{
Martine Gross, Laurence Brunet, Michelle Giroux
}

A la différence du Canada où la gestation pour autrui (GPA) est légale, y compris au Québec même si dans cette province, le contrat de GPA est nul et ne peut avoir de valeur exécutoire (Art. 541 C.c.Q. ; Loi sur la procréation assistée 2004, art. 6; Giroux 2011 ; Moore 2013)', en France, le recours à la GPA est strictement prohibé. Malgré cela, des couples hétérosexuels et des couples gays, ont recours à la GPA en se rendant dans les pays où elle est légale. Lorsqu'ils reviennent en France, la transcription de l'acte de naissance de l'enfant au service central de l'état civil, nécessaire pour reconnaître le lien de filiation à l'égard des parents d'intention, est incertaine. Plusieurs cas de figure se présentent selon que les parents d'intention sont mentionnés ou non sur l'acte de naissance étranger et selon que cette mention figure à la suite d'un jugement étranger d'adoption par le conjoint ou la conjointe du père.

Dans ce chapitre, nous nous intéressons aux déclarations des juges français concernant l'établissement de la filiation d'un enfant né du recours à une GPA. Quels sont les arguments mobilisés par les juges pour accepter ou refuser d'établir la filiation d'un enfant issu d'une pratique prohibée en France ? Le recours à la GPA est-il considéré comme une fraude à la loi suffisante pour empêcher de relier l'enfant à ses parents d'intention ? L'intérêt de l'enfant constitue-t-il un argument suffisant pour au contraire établir sa filiation ?

Nous nous appuierons pour cela sur l'analyse d'une partie des matériaux d'un projet de recherche international (France, Québec, Belgique et Espagne) et pluridisciplinaire (droit et sciences sociales), réalisé avec le soutien de la Mission Droit \& Justice du ministère de la justice en France. Ce projet de recherche s'intéresse aux situations où des Français recourent à des techniques de reproduction assistées telles que l'insémination artificielle (IA) ou la fécondation in vitro (FIV) avec don de gamètes ou la GPA - le plus souvent avec recours à

\footnotetext{
'Le présent chapitre porte sur le droit français. Cependant, bien que nous ayons jugé utile d'effectuer quelques précisions sur le droit québécois au passage, les références à ce dernier ne sont toutefois pas exhaustives.

${ }^{2}$ Nous remercions vivement la Mission de recherche Droit et Justice en France pour le financement accordé à ce projet de recherche copiloté par Michelle Giroux (Université Ottawa) et Jérôme Courduries (Université Jean-Jaures, Toulouse). Pour plus de détails sur le projet, voir le lien suivant: http://www.gip-recherche-justice.fr/publication/le-droit-a-lenfant-et-lafiliation-en-france-et-dans-le-monde-2/
} 
une donneuse d'ovocyte différente de la femme porteuse - à l'étranger et entendent voir reconnaître leur configuration familiale grâce à une transcription à l'état civil français de l'acte de naissance étranger ou via l'adoption de l'enfant du conjoint.

Dans le cadre de ce projet, des entretiens ont eu lieu avec des familles homo et hétéroparentales ainsi qu'avec des magistrats et des avocats. Les entretiens avec les magistrats avaient pour objectif d'explorer les arguments mobilisés et leurs interrogations lorsqu'ils sont sollicités pour établir la filiation d'un enfant conçu à l'issue du recours à l'étranger à une assistance médicale à la procréation (AMP) ou à une GPA.

Pour contacter les magistrats français, nous avons sollicité auprès de la Mission de recherche Droit et Justice une lettre de soutien attestant l'intérêt de la recherche et le financement accordé par le GIP Mission de recherche Droit et Justice. Cette lettre a été envoyée à 36 tribunaux de grande instance (TGI), 1 Cour d'appel et 36 parquets ${ }^{\varsigma}$ Lorsque des réponses favorables nous sont arrivées (12 TGI, 11 parquets) nous avons envoyé aux présidents de TGI et de Cour d'appel, ainsi qu'aux procureurs de ces juridictions une lettre présentant de manière synthétique les objectifs de la recherche afin de solliciter leur accord pour contacter les magistrats en charge des affaires, ainsi que leurs noms et coordonnées. Nous avons ensuite écrit, après accord des Procureurs et des Présidents de TGI ou de cours d'appel, aux magistrats concernés. 22 magistrats ont finalement donné leur accord. À ce jour, une série d'entretiens a été réalisée auprès de 16 magistrats français (13 femmes, 3 hommes): 14 siégeaient dans un TGI (9 juges du siège, 5 procureurs, vice-procureurs ou substituts), un substitut du procureur dans une Cour d'appel et un conseiller à la Cour de cassation. Cinq juges siégeaient à Paris ou en région parisienne, les autres ailleurs en France dans diverses régions. Leurs parcours professionnels au sein de la magistrature sont diversifiés. La plupart ont exercé dans plusieurs juridictions et dans des fonctions variées, notamment juge pour enfant, juge aux affaires familiales. Ils ont en moyenne 12 ans d'expérience professionnelle.

\footnotetext{
' En France, le terme «conjoint» désigne l'époux ou l'épouse. En droit civil québécois, le terme signifie les membres du couple mariés ou unis civilement. L'expression québécoise « conjoint de fait » n’est pas utilisée en France, on parle alors de concubin.

${ }^{4}$ Dans le cadre de ce projet de recherche, nous nous sommes aussi intéressés à la situation des couples de femmes françaises ou franco-canadiennes installées au Québec qui souhaitent voir reconnaître en France la double filiation maternelle reconnue dans l'acte de naissance au Québec, ou encore à la perplexité des juges français sollicités pour une adoption de l'enfant de la conjointe lorsque l'enfant est né du recours à un donneur de sperme connu. Dans une moindre mesure, quelques entretiens ont aussi eu lieu au Québec avec des juges et des avocats québécois sur les arguments qu'ils mobilisent lorsqu'ils sont sollicités pour l'adoption de l'enfant du conjoint ou de la conjointe lorsque l'enfant est né du recours à une GPA.

s Pour plus de précisions sur le fonctionnement de la justice civile en France, voir la prochaine section.

"Les procureurs, dits juges du «parquet», représentent le ministère public, l'autorité chargée de défendre l'intérêt de la société et la bonne application de la loi.
} 
Pour respecter l'anonymat des personnes interrogées, nous avons attribué un numéro à chaque TGI pour éviter d'avoir à nommer sa ville de rattachement. Le cas du parquet de Nantes fait exception à cause de son rôle particulier dans les demandes de transcription des actes de naissance délivrés à l'étranger.

Si le prononcé de l'adoption de l'enfant du conjoint entre dans les compétences des juges de TGI, il n'en va pas de même de la reconnaissance en France de l'acte de naissance d'un enfant né à l'étranger d'une GPA. La transcription d'un acte étranger de naissance sur les registres français de l'état civil est facultative, mais l'absence de transcription prive l'enfant de la jouissance au quotidien des droits attachés à la filiation. L'obtention d'un livret de famille nécessite notamment cette transcriptions. La compétence en est exclusivement dévolue au service central d'état civil de Nantes, créé en 1965 et dépendant du Ministère des Affaires étrangères. Ce service est en effet chargé de l'état civil des Français nés à l'étranger, ainsi que du recueil et de la transcription des actes civils faits par des Français à l'étranger.

Lors des entretiens, les juges de TGI ont été interrogés sur la question de prononcer ou non l'adoption de l'enfant de la conjointe en cas de recours à une insémination artificielle avec donneur de sperme (IAD) à l'étranger et également sur leur point de vue au sujet de la transcription d'un acte de naissance établi à l'étranger pour un enfant né à l'étranger grâce à une GPA, même s'ils ne peuvent être saisis d'une telle demande qui, comme on l'a dit, ne fait pas partie de leurs attributions. Même si les réflexions que les juges nous ont confiées décrivent des situations juridiques parfois virtuelles quand il s'agissait de la transcription de l'acte de naissance, il n'en reste pas moins qu'elles peuvent nous permettre de découvrir les arguments mobilisés en faveur, ou contre, l'établissement de la filiation d'un enfant issu de pratiques procréatives prohibées en France.

\section{Fonctionnement de la justice en France}

Rappelons brièvement le fonctionnement de la justice civile en France. La majorité des affaires peut être jugée deux fois au fond, une première fois par le juge dans un tribunal d'instance ou de grande instance (TGI), une deuxième fois, si l'une des parties conteste la décision en première instance, par le juge d'appel dans une cour d'appel. Ensuite l'arrêt d'appel peut éventuellement faire l'objet d'un pourvoi devant la Cour de cassation afin de

\footnotetext{
'Lors de l'entretien, nous avons obtenu le consentement du procureur de Nantes d'utiliser le contenu de l'entretien, même si l'anonymat ne pouvait être assuré.

- Comme la Cour d'appel de Rennes a plusieurs fois eu l'occasion de le rappeler. Voir par exemple, la décision du 27 juin 2016 (6è ch A) ou celle du 12 décembre 2016 (6è ch A).
} 
faire censurer sa non-conformité aux règles de droit. Les magistrats des tribunaux d'instance et de grande instance ainsi que ceux des cours d'appel sont les juges du siège (ceux qui jugent) et les procureurs ou leurs substituts sont les juges du parquet. Pour l'exemple, l'épouse d'une mère statutaire" peut présenter au TGI une requête en adoption plénière" de l'enfant de sa conjointe. Si le juge du TGI refuse de prononcer l'adoption, la requérante peut faire appel. Si la Cour d'appel valide la décision du juge de première instance, la requérante peut se pourvoir en cassation si elle estime que la décision résulte d'une mauvaise interprétation du droit (art 604 Code de procédure civile). Autre exemple, le service central de l'état civil à Nantes accepte de transcrire un acte de naissance établi à l'étranger pour un enfant dont les parents sont suspectés d'avoir eu recours à une GPA. Le procureur, responsable du service d'état civil, introduit un recours contentieux devant le TGI de Nantes. Si ce dernier confirme la décision de transcription, le procureur pourra faire appel de cette décision devant la Cour d'appel de Rennes. Si la Cour d'appel infirme la décision initiale, les requérants pourront se pourvoir en cassation. Si elle casse l'arrêt d'appel, la Cour de cassation ne pourra pas, sauf exception", reconsidérer les faits et devra renvoyer devant une autre juridiction de même nature. La juridiction de renvoi conserve cependant son entière liberté de décision et n'est pas obligée de rendre une décision conforme à celle de la Cour de cassation sur le point de droit censurés.

La Cour de cassation peut par ailleurs exercer une fonction consultative. Les tribunaux peuvent ainsi interpeller la Cour de cassation pour avis lorsqu'ils sont saisis d'une demande « soulevant une question de droit nouvelle, présentant une difficulté sérieuse, et se posant dans de nombreux litiges » (art. L. 441-1 et s. du Code de l'organisation judiciaire). La

\footnotetext{
' Il faut bien comprendre que la Cour de cassation est juge des jugements et non pas juge des affaires, juge du droit et non pas juge du fait. Elle ne peut ainsi revenir sur l'appréciation des faits de l'affaire qui sont tenus pour acquis devant elle et, en conséquence, elle ne pourra pas par exemple ordonner une mesure d'instruction. La Cour de cassation n'est donc pas un troisième degré de juridiction, «même si elle contrôle le raisonnement par lequel les juges du fond ont fait application de la règle de droit aux faits de l'espèce qui lui étaient soumis » (Cadiet et Jeuland, $2016: 713$ ).

${ }^{10}$ A l'instar de Virginie Descoutures (2008), nous préférons l'utilisation de « statutaire » plutôt que « légal » pour désigner le statut légal d'un parent, son lien de parenté étant reconnu juridiquement, pour éviter de supposer qu'un parent ne possédant pas ce statut serait « illégal ».

" L'adoption «plénière» substitue la filiation adoptive à la filiation de naissance (art 356 C. civ.). L'adoption «simple» ajoute la filiation adoptive à la filiation de naissance (art. 363 C. civ.) Au Québec, dans l'état actuel du droit, seule l'adoption "plénière » est possible, conformément à l'art. 577 C.c.Q.

${ }_{12}$ La loi de modernisation de la justice du XXIe siècle vient néanmoins d'étendre le champ des exceptions en prévoyant qu'après avoir cassé et annulé la décision qui lui est déférée la Cour de cassation pourra, « en matière civile, statuer au fond lorsque l'intérêt d'une bonne administration de la justice le justifie » (art. L. 411-3 Code de l'organisation judiciaire).

${ }_{13}$ Toutefois, lorsque se pose une question de principe ou en cas de second pourvoi contre l'arrêt rendu par la Cour d'appel de renvoi, la Cour de cassation statuera de manière solennelle en assemblée plénière. Si l'assemblée plénière de la Cour de cassation casse l'arrêt rendu par la première Cour d'appel de renvoi, alors la seconde Cour d'appel de renvoi sera tenue de se conformer à la solution de droit dégagée par la Cour de cassation.
} 
finalité de cette procédure est de prévenir le contentieux susceptible de naître de l'interprétation incertaine d'une norme nouvelle. C'est cette procédure qui a été mobilisée en France, à la suite de la loi du 17 mai 2013 autorisant le mariage entre personnes de même sexe, lorsque les décisions concernant, au sein des couples lesbiens, l'adoption de l'enfant de la conjointe divergeaient d'un tribunal à l'autre. Certains tribunaux interprétaient que le recours à l'AMP à l'étranger constituait une fraude à la loi de nature à faire obstacle au prononcé de l'adoption, puisqu'en France l'AMP n'est autorisée exclusivement qu'aux seuls couples constitués d'un homme et d'une femmes. D'autres tribunaux au contraire ne se préoccupaient pas de la manière dont avait été conçu l'enfant pour lequel l'adoption de l'enfant de la conjointe était requise (Brunet, 2015). Plusieurs tribunaux ont sollicité la Cour de cassation pour un avis sur cette question. Deux avis identiques ont été rendus en

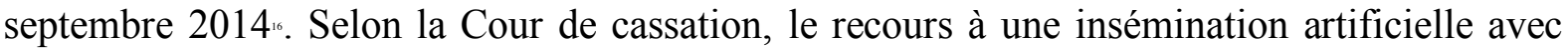
don de sperme anonyme à l'étranger ne fait pas obstacle au prononcé de l'adoption de l'enfant de la conjointe. La plupart des tribunaux se sont rangés depuis lors à cet avis même si celui-ci, par nature, ne lie ni la juridiction qui a formulé la demande d'avis, ni a fortiori les autres juridictions.

Lorsqu'une personne a épuisé tous les recours légaux dans son pays sans avoir obtenu satisfaction, elle peut déposer une requête devant la Cour européenne des droits de l'homme $(\mathrm{CEDH})$ si elle peut prouver qu'un de ses droits fondamentaux a été atteint.

\section{La filiation}

Sur le plan strictement juridique, l'établissement d'un lien de filiation entre un enfant et un parent d'intention soulève deux difficultés différentes selon qu'il s'agit d'une mère d'intention ou d'un père d'intention".

En droit français, la filiation maternelle repose sur le principe mater semper certa est : la femme qui accouche est la mère. Aucune démarche n'est requise de sa part pour faire établir juridiquement son lien de maternité à l'égard de l'enfant, la seule mention de son nom

\footnotetext{
${ }^{14} \mathrm{La}$ « fraude à la loi » est un contournement intentionnel de la loi française. Quand elle est constatée par les juges, elle prive du bénéfice de l'application du droit français celui qui l'a initialement mise en œuvre, pour tous les effets découlant de cette fraude.

${ }^{15}$ Art. .L. 2141-2 du Code de la santé publique.

${ }^{16}$ Avis ${ }^{\circ} 15010$ et $\mathrm{n}^{\circ} 15011$ du 22 septembre 2014.

"Dans la suite de ce chapitre, nous désignerons par mère d'intention, la femme qui n'a pas porté son enfant mais qui a souhaité en être la mère statutaire aux côtés de son compagnon, géniteur de l'enfant; nous désignerons par père d'intention l'homme, qui a souhaité être le père statutaire de son enfant aux côtés de son compagnon, géniteur ou père statutaire de ce dernier. Les familles rencontrées dans le cadre de ce projet sont des couples de femmes qui ont eu recours à un don de sperme, des couples d'hommes ou des couples hétérosexuels qui ont eu recours à une GPA sans don de sperme avec ou sans don d'ovocyte.
} 
sur l'acte de naissance de l'enfant y suffit (art. 311-25 du Code civil). Autrement dit, sauf en cas d'adoption, une femme qui n'a pas accouché son enfant ne peut en être la mère. Par ailleurs, un autre principe, celui de l'exclusivité de la filiation, préside à son établissement. Un enfant ne peut avoir qu'un seul père et une seule mère (art. 320 du Code civil). La loi du 17 mai 2013 en ouvrant le mariage aux personnes de même sexe, a autorisé l'adoption d'un

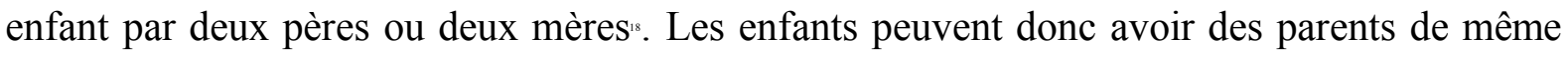
sexe. Cependant, un enfant ne peut pas avoir deux pères ou deux mères, dès sa naissance (art 6-1 du Code civil). Un enfant peut avoir deux parents de même sexe soit parce qu'il a été adopté par un couple marié de même sexe soit parce qu'il a fait l'objet d'une adoption par le conjoint de son père ou par la conjointe de sa mère. Ces derniers ne peuvent utiliser la reconnaissance par acte authentique (reçu par un officier de l'état civil ou par un notaire) ni la présomption de paternité liée au mariage.

L'acte de naissance établi à l'étranger des enfants nés du recours à la GPA diffère selon le pays où la femme porteuse a accouché. Dans certains états des Etats-Unis, le parent d'intention figure sur l'acte de naissance dès la naissance de l'enfant, en vertu d'un jugement préalable à la mise en œuvre de la GPA. Dans d'autres états des Etats-Unis ainsi que dans d'autres pays, tels que le Royaume Uni, la gestatrice, qu'elle soit ou non génétiquement liée à l'enfant, et le père figurent sur un premier acte de naissance, puis la gestatrice renonce à ses « droits parentaux » et le parent d'intention devient père ou mère en vertu d'un Parental Order $^{\prime \prime}$ ou par adoption de l'enfant du conjoint. Enfin dans certains pays et états des EtatsUnis, le père biologique peut apparaître seul sur l'acte de naissance. La mère ou le père d'intention devront faire postérieurement une démarche pour obtenir un statut légal de parent ultérieurement.

La reconnaissance d'un acte de naissance étranger qui mentionnerait, à côté de la mère de naissance ou du père biologique, une mère d'intention ou un père d'intention pose donc une difficulté majeure pour le droit français de la filiation car elle établirait un lien de filiation soit envers une femme qui n'a pas porté l'enfant, soit envers deux pères, mais sans passer par le prononcé d'une adoption.

\footnotetext{
${ }^{18}$ L'adoption est ouverte depuis 1966 aux personnes seules mais l'adoption d'un enfant par un couple n'est possible que le si le couple est marié (art. 346 C. civ.).

"Parental order peut être traduit par « déclaration judiciaire de parenté ».
} 


\section{La situation juridique en France}

En France avant même que la loi ne vienne condamner les conventions de gestation pour autrui, les juges en ont sanctionné la pratique, au nom de la violation tant du principe d'ordre public de l'indisponibilité du corps humain que celui de l'indisponibilité de l'état des personnes ${ }^{x}$, et ont refusé de prononcer l'adoption de l'enfant né grâce à une femme porteuse par la mère d'intention, au motif que l'opération constituait un détournement de l'institution de l'adoption". En 1994 fut ensuite introduit dans le Code civil l'article 16-7 qui dispose que « toute convention portant sur la procréation ou la gestation pour le compte d'autrui est nulle ». L'article 227-12 du code pénal sanctionne la provocation à l'abandon d'enfant, l'entremise en vue de l'adoption ou en vue de la gestation pour le compte d'autrui. L'article 227-13 du code pénal sanctionne l'atteinte à l'état civil d'un enfant, par exemple en prétendant qu'il a pour mère une autre femme que celle qui l'a accouché. D'autres articles font rempart plus spécifiquement contre le développement de pratiques commerciales de GPA, tel l'article 16-1 du code civil qui dispose que « le corps humain, ses éléments et ses produits ne peuvent faire l'objet d'un droit patrimonial ».

Le droit positif n'a pas été modifié depuis. Certes un groupe de travail du Sénat consacré à la maternité pour autrui s'est prononcé en 2008 en faveur d'un encadrement légal de la GPA en France (Sénat, 2008). Mais la même année, l'office parlementaire d'évaluation des choix scientifiques et technologiques s'opposait à la levée de la prohibition (OPECST, 2008). En 2009, dans le cadre de la révision des lois de bioéthique, le Conseil d'État a rendu un avis préconisant le maintien de l'interdiction, tout en proposant que la situation juridique des enfants nés à l'étranger par recours à cette pratique soit aménagée, de façon que ceux-ci ne soient pas pénalisés par le fait que leurs parents d'intention ont eu recours à une pratique

\footnotetext{
${ }^{20}$ Le principe de l'indisponibilité de l'état des personnes signifie que nul ne peut par sa seule volonté se dépouiller, même à titre gratuit, des éléments qui constituent son identité civil (sexe, prénom, nom, filiation, nationalité, situation de famille...). Le principe de l'indisponibilité du corps indique que le corps humain est hors du commerce : il ne peut faire l'objet de transactions, que ce soit à titre onéreux ou à titre gratuit (Terré et Fenouillet, 2012: 72-73 et 147-148). On peine toutefois à découvrir l'existence de ces principes dans la tradition juridique française et, pour certains auteurs, la Cour de cassation a fait ici un usage incantatoire de ces principes, les « projetant de l'imaginaire dans le droit positif» (Gobert, 1992 ; Depadt-Sebag, 2004). Pour en prendre la mesure, il suffit de mentionner des pratiques, depuis longtemps admises en droit français, comme le don de lait, de sang, d'organes, de moelle, de gamètes, les recherches biomédicales auxquelles des personnes en bonne santé peuvent se prêter contre une indemnisation, etc.

${ }^{2}$ Cass., ass. plén., 31 mai 1991, D. 1991. Jur. 417, rapp. Y. Chartier et note D. Thouvenin ; Cass. $1^{\text {re }}$ civ., 9 déc. 2003 , D. 2004, p. 1988, note E. Poisson-Drocourt ; Cass. ${ }^{\text {re }}$ civ., 23 avril 2003, Dt famille 2003, 143, obs. P. Murat. Au Québec, deux courants jurisprudentiels ont coexisté, l'un permettant l'adoption, par consentement spécial (art. 555 C.c.Q.) au nom de l'intérêt de l'enfant, l'autre l'interdisant au nom de l'ordre public (art. 541 C.c.Q.), mais la Cour d'appel du Québec a tranché en faveur de l'adoption au nom de l'intérêt de l'enfant en cas d'exécution volontaire de la convention de GPA (Adoption -- 1445, 201 QCCA 1162; Adoption -- 161, 2016 QCCA 16. Pour des commentaires de ces jugements, voir notamment A.-M. Savard (2015) ; L. Langevin (2015) et Malaket (2015).
} 
interdite en France (Conseil d'État, 2009). En effet, si l'arsenal juridique avait dissuadé les couples de recourir en France à la GPA, la pratique n'avait pas pour autant disparu, elle s'était déplacée à l'étranger, dans les pays autorisant la GPA et ne la réservant pas aux ressortissants nationaux. Malgré cette délocalisation, l'intensité de la lutte du droit français contre de la GPA n'a pas diminué.

\section{L'état civil des enfants nés à l'étranger du recours à la GPA}

\section{Le retour en France des enfants issus d'une GPA réalisée à l'étranger se heurte à plusieurs} obstacles.

Le premier est celui de leur entrée sur le territoire français. Le lieu de la GPA a une importance non négligeable concernant la nationalité de l'enfant et la possibilité de le faire revenir en France. Dans les pays qui accordent la nationalité en vertu du droit du sol, par exemple, les Etats-Unis et le Canada, les enfants obtiennent la nationalité du pays où ils ont $\mathrm{vu}$ le jour et un passeport pour rentrer en France avec leurs parents. Dans les pays ne pratiquant pas le droit du sol, par exemple l'Inde ou l'Ukraine, les enfants dont les parents sont suspectés par les autorités françaises d'avoir eu recours à la GPA, n'obtiennent ni la nationalité française ni la nationalité du pays où ils sont nés. Ils ne peuvent rentrer en France à moins d'obtenir un laisser-passer consulaire, que les autorités administratives sont tenues de délivrer dans l'intérêt supérieur de l'enfant (Conseil Etat, réf., 3 août 2016, n 401924).

Le second obstacle concerne le droit international privé et porte sur la reconnaissance en France des liens de filiation, établis à l'étranger, des enfants à l'égard de leurs parents d'intention. En effet, pour éviter tout encouragement à recourir à la GPA, la France a jusqu'à très récemment refusé de transcrire les actes de naissance établis à l'étranger pour les enfants nés de cette pratique, alors même que depuis 2002, les tribunaux étaient saisis à plusieurs reprises de telle demandes.

Ainsi, la Cour d'appel de Paris avait refusé le 25 octobre 2007 que la transcription dans les registres français d'état civil du Service central d'état civil de deux actes de naissance américains puisse faire l'objet d'une demande d'annulation de la part du Ministère public, au nom « des conséquences contraires » que cette annulation aurait sur « l'intérêt supérieur des enfants ». Les actes de naissance transcrits rattachaient alors les enfants, deux jumelles, à leur géniteur (père biologique) et leur mère intentionnelle. Cependant, cet arrêt avait été cassé par la Cour de cassation le 17 décembre 2008, au motif de l'intérêt à agir du ministère public pour demander la nullité de la transcription sur les registres français de l'état civil des actes de 
naissance des enfants nés d'une gestation pour autrui à l'étranger.

Le 6 avril 2011, au nom de la contrariété à l'ordre public international français et au principe de l'indisponibilité de l'état des personnes, « principe essentiel du droit français », la Cour de cassation, dans trois arrêts de principe (dont l'un était la suite de l'affaire Mennesson ayant déjà donné lieu à l'arrêt du 17 décembre 2008), avait refusé « de faire produire effet, au regard de la filiation, à une convention portant sur la gestation pour le compte d'autrui ». Elle a ainsi confirmé le refus de transcription des actes de naissance étrangers des enfants établissant la filiation à l'égard de leur père biologique et de leur mère d'intention. Deux des couples concernés (Mennesson et Labassée) par ces arrêts ont saisi la Cour européenne des droits de l'homme.

La Cour d'appel de Rennes, dans un arrêt rendu en date du 21 février 2012, a confirmé un jugement du Tribunal de grande instance de Nantes, du 17 mars 2011, qui avait ordonné la transcription de l'acte de naissance d'un enfant né à l'étranger, supposément par GPA ${ }^{2}$. Le ministère public s'est alors pourvu en cassation.

Une étape supplémentaire a alors été franchie dans l'intensité de la lutte juridictionnelle contre les recours à la GPA à l'étranger : dans ses deux arrêts du 13 septembre 2013 (cassant, pour l'un, l'arrêt de la CA Rennes du 21 févier 2012 précédemment mentionné) et dans celui du 19 mars 2014, la Cour de cassation avait substitué l'argument de la fraude à la loi à celui de la contradiction avec l'ordre public français pour refuser la transcription de l'acte de naissance indien d'un enfant né par GPA où figuraient le nom de la mère de naissance et celui de son père géniteur. La gravité de la fraude à la loi neutralisait, selon la Cour de cassation, toute considération de l'intérêt de l'enfant ou du respect de sa vie privée. De plus, la Cour s'était prévalue de la fraude à la loi pour valider l'annulation de la reconnaissance de paternité que le père biologique avait faite en France.

En juin 2014, dans les arrêts Mennesson et Labassée c/ France, la Cour européenne des droits de l'homme (CEDH) a condamné la France en considérant que le refus de la transcription des actes de naissance des enfants avait porté atteinte à « leur identité au sein de la société française $w^{3}$. Aux yeux des juges européens les conséquences juridiques de la non reconnaissance en droit français du lien de filiation entre les enfants et les parents d'intention

\footnotetext{
22 Cour d'appel de Rennes (Ch 6 A), 21 février 2012, LexisNexis n 434, 11/02758.

${ }^{23}$ CEDH 26 juin 2014, $n^{\circ}$ 65192/11, Mennesson c/ France et n 65941/11 Labassée c/ France.
} 
« affectaient significativement le droit au respect de la vie privée qui implique que chacun puisse établir la substance de son identité, y compris sa filiation ». Se posait donc là « une question grave de compatibilité de la situation avec l'intérêt supérieur des enfants dont le respect doit guider toute décision les concernant "(§ 99). La CEDH a souligné que cette préoccupation « prenait en relief particulier » lorsque, comme en l'espèce, le père biologique se voyait privé de toute possibilité d'établir sa paternité à l'égard des enfants (§ 100).

Le 3 juillet 2015, suite à la condamnation de la France par la CEDH, la Cour de cassation a confirmé, par deux arrêts, l'obligation faite au Service de l'état civil d'inscrire les actes de naissance du moment que ceux-ci «n'étaient ni irréguliers ni falsifiés et que les faits qui y étaient déclarés correspondaient à la réalité (article 47*2) ». En juillet 2015, la Cour de cassation a donc opéré un revirement de jurisprudence pour se mettre en conformité avec la décision de la $\mathrm{CEDH}$, en entérinant que la théorie de la fraude à la loi ne pouvait plus faire échec à la transcription de l'acte de naissance. Pourtant elle n'a fait ici qu'une application $a$ minima des décisions de la CEDH qui s'imposaient à elle, dans la mesure où la question de la transcription de la filiation établie à l'étranger à l'égard du parent d'intention (la mère d'intention dans un couple de sexe différent ou le second père dans un couple d'hommes) n'était pas soulevée dans les espèces qui lui étaient soumises. Elle a pris soin de souligner dans son communiqué de presse qu'elle ne s'était pas prononcée sur ce cas de figures.

En résumé, voici ce que l'on peut dégager de cette évolution jurisprudentielle. Avant les arrêts de la Cour de cassation du 3 juillet 2015 qui ont opéré un revirement partiel de la jurisprudence, pour être en conformité avec les décisions de la CEDH du 26 juin 2014, le recours à la GPA, en tant que fraude à la loi, faisait obstacle à la transcription à l'état civil français de l'acte de naissance dressé à l'étranger.

Depuis juillet 2015, la transcription de l'acte de naissance est donc possible si celui-ci est conforme à la « réalité » (article $47 \mathrm{C}$. civ). Au juge d'interpréter ce que recouvre le terme « réalité » au sens de l'article 47. Il peut s'agir de la « réalité juridique » et il s'agira alors de vérifier si l'acte de naissance étranger a été établi de manière authentique sans frauder la loi

\footnotetext{
${ }^{24}$ Article 47 : « Tout acte de l'état civil des Français et des étrangers fait en pays étranger et rédigé dans les formes usitées dans ce pays fait foi, sauf si d'autres actes ou pièces détenus, des données extérieures ou des éléments tirés de l'acte luimême établissent, le cas échéant après toutes vérifications utiles, que cet acte est irrégulier, falsifié ou que les faits qui y sont déclarés ne correspondent pas à la réalité ».

${ }^{2}$ https://www.courdecassation.fr/jurisprudence_2/communiques_presse_8004/archives_6783/2015_8003/civil_enfants_3223 7.html.
} 
du pays où il a été dressé. Mais il peut s'agir aussi de la « réalité biologique ». Nous nous sommes entretenus avec le procureur de Nantes qui contrôle, entre autres, toutes les demandes de transcription d'actes de naissance étrangers. Il interprète la « réalité » de l'article 47 comme la réalité «biologique », c'est-à-dire qu'il accepte de reconnaître la filiation paternelle du géniteur, mais pas la parenté d'intention. Un acte de naissance mentionnant la mère d'intention ou le deuxième père, à côté du nom du père biologique, ne serait pas transcrit en totalité ${ }^{2}{ }^{2}$. En revanche, si la parenté d'intention a été instituée dans un deuxième temps, à la suite d'une adoption prononcée par un juge étranger conformément à la législation locale et une fois recueilli expressément le consentement à l'adoption de la femme qui a porté l'enfant, l'acte de naissance pourrait être transcrit. Par ce raisonnement, le principe selon lequel la mère est toujours celle qui a accouché serait sauf et le parent d'intention ne se substituerait à la mère de naissance sur l'acte de naissance que par une opération reconnue du droit, l'adoption, et non au terme de ce qui, sinon, pourrait s'apparenter à une simulation sur l'état civil, pénalement répréhensible (art. 227-13 du code pénal).

D'autres interrogations demeurent encore à la suite les arrêts Mennesson et Labassée. En effet lorsque les juridictions françaises ont rendu une décision définitive annulant ou refusant la transcription des actes de naissance étrangers sur les registres français, une nouvelle demande se heurterait à l'autorité de la chose jugée, règle fondamentale de la procédure civile française qui interdit aux parties de saisir à nouveau le juge une fois que le contentieux a été définitivement tranché. C'est pourquoi les actes de naissance des enfants Mennesson n'ont toujours pas été transcrits sur les registres français de l'état civil. Ces blocages liés à l'autorité de la chose jugée sont à l'origine d'une nouvelle condamnation de la France par la CEDH dans les arrêts Foulon et Bouvet du 21 juillet 2016, et plus implicitement, dans l'arrêt Laborie du 19 janvier 2017'. Le législateur français a alors saisi l'occasion du chantier législatif sur la modernisation de la justice du $\mathrm{XX}^{\mathrm{e}}$ siècle pour venir

\footnotetext{
${ }_{26}^{26}$ Pour une application, voir CA Rennes (ch 6 A), 7 mars 2016 ( $\left.n^{\circ} 178,15 / 03855\right)$.

${ }^{27}$ Ces critères pris en compte par le procureur de Nantes pour autoriser ou non la transcription ont conduit à une situation tout à fait paradoxale dans le cas d'un couple de femmes françaises vivant au Québec. Ces femmes y ont mis au monde deux enfants issus du recours à une AMP. Les enfants ont un acte de naissance mentionnant qu'ils ont pour mères les deux femmes selon le droit québécois (Art. 115 C.C.Q). La transcription de ces actes de naissance leur a été refusée par le Consulat de France au motif qu'ils comportaient deux mères et que seule la mère biologique pourrait être reconnue. La mère qui n'a pas porté l'enfant a déposé une requête au TGI de Paris pour adopter les enfants de sa conjointe comme le permet la loi du 17 mai 2013. Le TGI de Paris a refusé de prononcer l'adoption des enfants au motif que la requérante était déjà la mère selon la filiation indiquée sur les actes de naissance étrangers (TGI de Paris, 1/5/1 Chambre du conseil $n^{\circ}$ RG : 15/18492, jugement du 7 décembre 2016).

${ }^{28}$ CEDH 21 juillet 2016, Foulon et Bouvet c/ France, $n^{\circ}$ 9063/14 et 10410/14.

${ }^{29}$ CEDH 19 janvier 2017, Laborie c/ France, n 44024/13.
} 
pallier cette lacune du droit français : la loi du 18 novembre 2016 est venue introduire la possibilité d'un réexamen, sous certaines conditions, d'une décision définitive, rendue en matière d'état des personnes, qui a donné lieu à une condamnation de la France par la CEDH.

Condamnée par la CEDH pour avoir porté atteinte à « l'identité des enfants » dont elle avait refusé de transcrire les actes de naissance, la France a donc été conduite à prendre davantage en compte l'intérêt de l'enfant. En conséquence le droit français se retrouve pris en étau entre le respect de l'intérêt supérieur de l'enfant, qui commande de transcrire l'acte de naissance valablement établi à l'étranger, et la crainte que cette possibilité de transcription n'encourage la pratique de la GPA, contraire à l'ordre public.

Voilà comment le procureur de Nantes analyse cette situation :

On arrive aujourd'hui à une contradiction majeure de notre droit positif qui est de dire: on ne change pas la loi nationale, [...] chaque État est souverain pour décider quelles sont les lois qui doivent gouverner telle question qui lui est essentielle comme la filiation en l'espèce, mais que l'état civil...on doit octroyer un état civil à ces enfants-là c'est la portée finalement de la CEDH ; mais on arrive à une contradiction majeure, c'est que le message qui est reçu par les personnes qui sont en mal d'enfant c'est: vous pouvez aller à l'étranger, vous ne risquez plus rien, ça devient possible et licite. Donc, on arrive à une justice à deux vitesses finalement, où les personnes [...] qui préfèrent la voie de l'adoption sont évidemment désavantagées si je puis dire, par rapport à ceux qui se moquent des interdits légaux et ont les moyens d'aller souscrire ce type de conventions à l'étranger.

Il souligne ainsi la double contradiction d'une part, entre le droit supranational qui impose de prendre en compte l'intérêt de l'enfant et la conception nationale du droit de la filiation ; d'autre part, entre la liberté laissée par la CEDH aux états membres de prohiber la GPA sur leur sol et l'impossibilité de sanctionner la transgression d'un tel interdit une fois que l'enfant est né. Enfin il dénonce l'inégalité entre les citoyens qui ont les moyens de se tourner vers la GPA et les autres.

\section{Les arguments mobilisés par les juges français}

Les juges français rencontrés lors des entretiens menés dans le cadre de notre projet de recherche, sans avoir eu à rendre des décisions en cette matière, se sont posés plusieurs 
questions : la GPA est-elle une fraude à la loi empêchant l'établissement de la filiation de l'enfant; peut-on se contenter d'établir la filiation paternelle biologique en négligeant la parenté d'intention; si la filiation ne peut être établie, ou si elle ne peut être établie que partiellement, cela constitue-t-il une atteinte à l'intérêt de l'enfant ?

\section{Considérer la GPA comme une fraude à la loi ou bien prendre en compte l'intérêt de} l'enfant?

Les recours à la GPA dans un pays qui l'autorise, y compris à des non ressortissants, constitue-t-il une «fraude à la loi » française ? C'est la question qui a été soulevée dans plusieurs jugements ${ }^{\text {si }}$ et par certains commentateurs (Chénedé, 1997 ; Fabre-Magnan, 2013b ; Mirkovic, 2013 ; Frison-Roche, 2014 ; Marais, 2015). Pour ce qui est de la transcription des actes de naissance à l'état civil français pour les enfants nés d'une GPA, la question continue à être posée par des acteurs du monde judiciaire. Si cette « fraude à la loi » est retenue, est-ce là pour autant un motif pour refuser de transcrire sur les registres français l'état civil de l'enfant valablement établi à l'étranger ? Si cette fraude n'était plus retenue, la reconnaissance de la filiation envers les parents à l'origine du projet d'enfant pourrait-elle être encore refusée? Si le recours à la GPA était considéré comme une fraude à la loi, l'intérêt de l'enfant à voir établie sa filiation à l'égard des parents qui ont souhaité sa mise au monde et qui l'élèvent doit-il toujours prévaloir ? La question même de l'intérêt de l'enfant, sa définition et ses contours font l'objet de discussions. Ces discussions mettent en lumière le hiatus important qui demeure entre préservation de l'ordre public et prise en compte de l'intérêt de l'enfant.

L'extrait d'entretien suivant en témoigne.

TGI 6, un juge du siège, 17 décembre 2015 :

La GPA pollue tout, c'est une fraude à la loi et donc ça interdit le prononcé [de l'adoption par le conjoint ou la conjointe du père], je ne suis pas sûre qu'il $y$ ait une modification de la jurisprudence depuis cette date, la seule chose c'est que maintenant on peut, si l'acte d'état-civil est formellement régulier à l'étranger, donner un acte d'état-civil français voilà. Moi je suis assez [pour] a priori, la jurisprudence de la Cour de cassation est assez claire, maintenant il faut régler la situation de ces enfants qui ne peuvent pas vivre sans papiers.

\footnotetext{
${ }^{30}$ Par exemple Cass. civ. $1^{\circ}, 13$ sept. 2013, pourvois n ${ }^{\circ} 12-30138$ et 12-18315; Cass. civ. $1^{\circ}, 19$ mars 2014 (13-50.005).
} 
Ce juge semble ainsi soulagé de pouvoir s'appuyer sur la jurisprudence pour tenir compte de l'intérêt de l'enfant et lui donner un état-civil français.

Une partie des magistrats rencontrés s'est néanmoins exprimée sur les risques de marchandisation du corps des femmes. L'extrait suivant illustre cette inquiétude.

TGI 7, Vice-procureur, 16 décembre 2015 :

C'est vrai que ce qui est problématique c'est que c'est devenu une source de profit dans certains pays étrangers au détriment des femmes qui se retrouvent exploitées, tel que ça se conçoit dans certains pays ça s'apparente à une nouvelle forme d'esclavage moderne ce qui me gêne prodigieusement.

Ces juges témoignent ainsi du fait qu'ils sont traversés par les mêmes arguments et objections qui ont cours dans le débat public autour de la GPA.

\section{Le débat autour de la GPA dans lequel s'inscrivent les interrogations des juges}

Les tenants et les opposants à l'encadrement juridique de la GPA sont d'accord sur un point, l'objectif est d'éviter la marchandisation du corps humain et l'exploitation des femmes. Ceux qui souhaitent maintenir la prohibition qui a cours actuellement en France exposent principalement deux arguments. Tout d'abord, la GPA serait contraire au principe de la dignité de la personne humaine car elle reviendrait à instrumentaliser le corps des femmes, à en faire un objet marchand. Ensuite, la GPA serait contraire à l'intérêt de l'enfant parce qu'elle constituerait un abandon à la naissance et compromettrait son épanouissement à venir.

Concernant le principe de la dignité de la personne humaine qui impose, entre autre, de ne pas traiter un être humain comme un bien que l'on peut acquérir, son contenu demeure flou et il n'a jamais été mobilisé en tant que tel par les juges français pour sanctionner les pratiques de GPA. En dépit de la pluralité des courants féministes, l'ensemble des féministes s'accordent pour dire que la grossesse choisie est une liberté individuelle, et même une « conquête » des femmes avec la légalisation de la contraception et la reconnaissance progressive d'un véritable droit à l'interruption volontaire de grossesse. Cependant, elles sont partagées sur la question de la GPA (Markens, 2007 ; Hammons, 2008). Les opposants à la GPA y voient un esclavage moderne" qui ne saurait être régulé (Frison-Roche, 2014). Certaines féministes comparent la GPA à la prostitution (Agacinski, 2013). Toutefois, une partie d'entre elles plaide pour la légalisation de la pratique (Iacub, 2004 ; Fouque, 2009 ;

${ }^{31}$ Voir http://www.lefigaro.fr/vox/societe/2015/06/25/31003-20150625ARTFIG00087-bernard-debre-gpa-vers-un-esclavagemoderne-des-femmes.php. C'est également un argument mis en avant par le mouvement "manif pour tous », voir http://www.lamanifpourtous.fr/actions-de-nos-regions/cp-la-gpa-est-un-nouvel-esclavage-qui-asservit-les-femmes/. 
Badinter, Théry et al., 2012). D'autres vont jusqu'à recommander la rémunération de la femme porteuse en compensation du travail qu'elle effectue (Borrillo, 2016; Tabet, 2014 ; Langevin, 2015) Selon certains auteurs, les dérives marchandes observées au niveau mondial ne constitueraient pas un contre-argument à l'autorisation de la GPA en France. Elles viendraient au contraire souligner le devoir de notre pays de se doter d'une loi qui ne se contenterait pas de repousser les difficultés hors de nos frontières (Nisand, 2010 ; Gross, 2014). Plusieurs pays dont le Royaume Uni, le Canada, la Grèce ou Israël ont légalisé la GPA en contrôlant strictement les sommes versées à la gestatrice pour la rembourser de tous les frais afférents à la grossesse, de manière à ce que la GPA soit pensée comme un don ${ }^{2}$. La politologue Jennifer Merchant expose ainsi quatre modèles de GPA « éthiques » en Floride, Utah, Washington et New Hampshire (Merchant, 2013). Cependant pour certains juristes, toute indemnisation suffit à effacer le caractère altruiste de la GPA (Fabre-Magnan, 2013a) tandis que d'autres auteurs voient dans la GPA une vente d'enfant qui ne saurait être encadrée (Agacinski, 2009 ; Mattei, 2011 ; Neirinck, 2015). Les trafics d'enfants n'ont pas abouti à l'interdiction de l'adoption mais ont conduit les pays à s'en prémunir au travers de l'élaboration de la convention internationale de la Haye (1993) sur la protection des enfants et la coopération en matière d'adoption internationale". C'est pourquoi certains auteurs préconisent l'élaboration d'une telle convention ${ }^{*}$ pour réglementer de manière acceptable la pratique de la GPA (Brunet, 2013 ; Trimming et Beaumont, 2013 ; Fulchiron, 2014).

La deuxième objection à la légalisation de la GPA soutient que cette pratique serait contraire à l'intérêt de l'enfant car elle équivaudrait à son abandon par la femme qui l'a porté (Busnel, Frydman et al., 2010). L'enfant a pourtant déjà des parents intentionnels sans lesquels il n'aurait jamais vu le jour. Il n'est donc pas abandonné, sans parent (Sénat, 2008 ; Nisand, 2010 ; Catto, M-X., 2013). Pour certains défenseurs de cet argument, la femme qui accouche d'un enfant en est toujours la mère, qu'elle soit ou non liée génétiquement à l'enfant, qu'elle prenne ou non soin de lui. Pour d'autres, les échanges intra-utérins, tant physiologiques que psychologiques, sont primordiaux dans les relations mère-enfant. Des travaux récents en épigénétique montrent que les interactions entre la gestatrice et le fœtus

\footnotetext{
${ }^{32}$ Au Canada, l'entrée en vigueur de l'article 12 de la Loi sur la procréation assistée 2004 prévoyant le remboursement des dépenses se fait toujours attendre.

${ }^{33}$ Convention du 29 mai 1993 sur la protection des enfants et la coopération en matière d'adoption internationale (https://www.hcch.net/fr/instruments/conventions/full-text/?cid=69).

${ }^{34}$ Voir notamment les travaux en cours de la Conférence de la Haye de droit international privé (http://www.irenekaufer.be/index.php?option=com_content\&view=article\&id=235:meres-porteuses-contes-de-fees-etrapports-de-pouvoir\&catid=1:articles-blog\&Itemid $=4$ ).
} 
modifient les gènes du fœtus (Académie de médecine, 2014 ; Bühler, 2016). Pendant les neuf mois de grossesse, le fœetus est dans un rapport fusionnel avec celle qui le porte : il s'habitue à son corps, à son odeur, à sa voix. Pour d'autres cependant, la rupture dont souffrirait l'enfant en ne retrouvant pas à la naissance les sons, les odeurs et les repères qu'il avait pendant la grossesse est toute relative (Henrion \& Bergoignan-Esper, 2009 ; Golombok et al., 2006). Les échanges intra-utérins ne sont pas déterminants au point que l'enfant aurait besoin, sous peine de traumatisme grave, de rester en contact intime avec la femme qui l'a porté. De plus, ces échanges dépendraient éminemment de chaque femme. D’autres encore remettent en question le principe mater semper certa est, car la grossesse ne serait pas tout (Bureau et Guilhermont, 2011). Certaines deviennent mères pendant la grossesse, mais d'autres ne s'attachent à l'enfant qu'une fois celui-ci né, d'autres encore, sous l'emprise avec un déni de grossesse, ignorent presque jusqu'au terme de leur grossesse qu'elles sont enceintes. Enfin, certaines femmes n'adopteront jamais l'enfant qu'elles ont mis au monde. L'existence de l'instinct maternel n'est pas prouvée, la gestation n'entraîne pas nécessairement l'émergence d'un tel sentiment (Badinter, 1980). René Almeling a enquêté auprès des donneuses d'ovocytes et des gestatrices aux États-Unis (Almeling, 2011). Les unes et les autres refusent de se considérer comme des mères, de sorte que leur geste ne peut s'interpréter comme un abandon.

\section{L'éthique internationale : deux poids deux mesures}

Pour en revenir aux discours des juges, plusieurs d'entre eux ont insisté sur l'ambivalence de la notion d'intérêt de l'enfant en droit international. Ils ont ainsi évoqué la Convention de la Haye relative à l'adoption internationale. Cette convention rédigée pour réguler la pratique de l'adoption dans l'objectif d'empêcher ou tout au moins de réduire les trafics d'enfants leur semble comparable à l'interdit de la GPA. Il s'agirait dans les deux cas d'éviter une commercialisation du corps humain. Ces juges ont comparé la transgression de l'ordre public qui consisterait à accepter de transcrire l'état civil des enfants nés du recours à la GPA et la transgression des conditions légales imposées par la Convention de la Haye dans l'adoption internationale. Dans le domaine de l'adoption, accorder l'adoption plénière, alors que le consentement de la mère de naissance n'est pas suffisamment éclairé ou semble vicié, serait un signal d'autorisation de transgresser les conditions légales. De même l'inscription à l'état civil français de l'acte de naissance d'un enfant issu de GPA, enverrait un signal d'autorisation tacite. 
L'extrait suivant illustre ce point.

TGI 4, un juge du siège, 12 novembre 2015 :

Une fois qu'on nous dit: l'intérêt de l'enfant c'est de vivre dans sa famille avec ceux qui l'élèvent c'est exactement la même chose que pour les adoptions internationales, avec des couples hétérosexuels mais qui ne respectent pas les conditions de l'adoption internationale...est-ce qu'on prononce l'adoption ou pas ? [...] mais après on sait bien que l'enfant il est là, c'est là où c'est une situation assez intenable, mais ce n'est pas propre aux couples de même sexe. Ces questions-là c'est compliqué parce qu'en fait si on voit l'intérêt quotidien de l'enfant, s'il [...] est pris en charge par des parents français qui vivent en France qui n'ont pas respecté la procédure qu'est-ce qu'on fait? "»

[...] ça veut juste dire qu'on ne respecte plus les conventions internationales, la convention de La Haye qui prévoit tout un tas de critères pour éviter que des adoptions se fassent dans des mauvaises conditions c'est-à-dire avec de l'argent qui transite par des intermédiaires, que l'enfant soit arraché à ses parents de façon pas très claire, si in fine au bout de la chaîne on dit oui, ça veut dire qu'on ne favorise pas l'adoption éthique. C'est des vraies questions...

[...] ça veut aussi dire qu'on ne permet pas à d'autres enfants d'avoir un processus éthique du ler coup. On est un peu pris entre le marteau et l'enclume, parce qu'à la fois il y a l'enfant, à la fois il y a des règles juridiques qui sont précises et qui sont établies en vue de permettre des adoptions éthiques.

[...] Ce qui est aussi compliqué, c'est que d'un côté on nous dit il y a eu fraude, et maintenant, aujourd'hui on nous dit : c'est l'intérêt de l'enfant soit, mais dès lors qu'on transcrit quelque chose qui à l'origine est une fraude au motif que c'est de l'intérêt de l'enfant, pourquoi est-ce que nous, [...] on continue à demander des signatures, des documents légalisés, etc...pour des adoptions internationales? [...]. Aujourd'hui au nom de l'intérêt de l'enfant on va transcrire l'état-civil issu de la GPA et nous pour des adoptions internationales, quel que soit le couple, s'il n'y a pas les consentements tamponnés, légalisés, si on n'a pas de certificat de conformité avec la convention de La Haye, on va dire qu'on ne prononce pas l'adoption. Alors on fait quoi finalement? 
La comparaison entre l'adoption internationale et la gestation pour autrui transnationale laisse les juges du siège des TGI perplexes. Les dernières décisions de la CEDH et de la Cour de cassation jettent en effet le trouble en validant indirectement des pratiques de gestation pour autrui bien moins réglementées que l'adoption internationale. A leurs yeux, ces décisions contribuent à disqualifier les strictes exigences posées par le droit international en matière d'adoption d'enfants étrangers et donnent une prime à ceux qui courtcircuitent ces exigences en recourant à la GPA.

Un juge exprime son dépit ainsi :

TGI 4, juge du siège, 12 novembre 2015 :

On est à la fois sur le respect de l'ordre public et à la fois sur l'intérêt de l'enfant ; à la fois sur le fait de promouvoir une éthique internationale, ne pas faire n'importe quoi et puis l'enfant il est là, et les arrêts de la cour de cassation et de la CDEH ça ne nous aide pas vraiment à y voir très clair.

$\mathrm{Si}$, pour les juges, l'intérêt de l'enfant ne semble pas être garanti de la même manière en droit international selon qu'est en cause la GPA ou l'adoption, la notion est par ailleurs obscurcie par des tensions sémantiques qui peuvent donner lieu à des appréciations contraires.

\section{L'intérêt de l'enfant : appréciation in concreto ou in abstracto ?}

La compréhension de l'intérêt de l'enfant fait l'objet de discussions. En quoi consistet-il exactement? Parle-t-on de l'intérêt de l'enfant, en général ? Ou bien parle-t-on de l'intérêt d'un enfant en particulier dans l'affaire qu'on a à juger? C'est toute la différence entre une interprétation in abstracto de l'intérêt de l'enfant, qui vise à protéger les enfants de toute forme de marchandisation et conduit à décourager les pratiques de gestation pour autrui, et une interprétation in concreto qui suppose, une fois l'enfant né, de sécuriser ses liens avec ses parents d'intention qui l'élèvent". Quoi qu'il en soit l'intérêt de l'enfant est façonné par les représentations sociales qui dépendent des circonstances et varient selon les lieux et les époques, comme le souligne une conseillère à la Cour de cassation dans un entretien du 23 mars 2016, dont voici un extrait :

${ }^{35}$ C'est l'argument défendu dans le contexte québécois par M. Giroux (2011) et B. Moore (2013), repris par la Cour d'appel du Québec dans l'arrêt Adoption -- 1445, 201 QCCA 1162. 
Aujourd'hui l'intérêt de l'enfant [...] ça peut toujours évoluer, c'est relativement modulable; en revanche sur la filiation [...] c'est pour la vie quoi, la responsabilité est immense et moi je comprends que mes collègues se posent des questions. Pour en revenir à la GPA, la Cour de cassation, c'est vrai, avait une vision in abstracto de l'intérêt de l'enfant pendant longtemps parce qu'elle considérait que c'était la vision du législateur français, [...]? L'idée c'est que pour le législateur français c'est un interdit fondamental. Pourquoi? parce qu'en fait derrière tout ça le législateur français considère que ce n'est pas l'intérêt des enfants en général d'être.....de faire l'objet d'une convention de gestation pour autrui pour être abandonné à la naissance par une femme en Inde ou ailleurs, et donc derrière c'est l'intérêt des enfants in abstracto et des mères porteuses qui a été pris en considération par le législateur français ; c'est d'ailleurs ce qu'a dit la CEDH en disant : le législateur français peut tout à fait interdire la GPA parce que derrière on prend en compte l'intérêt des enfants in abstracto et l'intérêt des mères porteuses. La Cour de cassation a eu cette analyse qu'on peut discuter tout à fait et dire: on prend l'intérêt in abstracto des enfants en général et donc c'est vrai ça le méconnaît peut-être in concreto mais c'est pour protéger ce qu'on considère comme n'étant pas souhaitable pour les enfants. [...] Aujourd'hui c'est différent puisque la CEDH nous dit: ce qui compte c'est l'intérêt de l'enfant in concreto, dans un dossier donné une fois que l'enfant est né, il n'est pour rien sur les circonstances de sa conception etc....donc in concreto il doit avoir un lien de filiation établi. Dont acte, très bien, maintenant c'est cette conception-là mais on peut aussi entendre que jusque-là la Cour de cassation s'abritait quand même derrière le législateur pour l'ordre public international français et donc un intérêt de l'enfant perçu in abstracto.

Même si la Cour européenne a clairement fait prévaloir une appréciation concrète de l'intérêt de l'enfant, l'embarras des juges paraît bien demeurer. Là où l'interprétation in abstracto de cette notion venait au soutien de l'ordre public français et consolidait les sanctions prononcées par les juges contre les pratiques de GPA, l'interprétation in concreto conduit à une divergence avec l'ordre public général qui semble entamer l'assurance du juge. C'est sur ce sentiment de fragilisation de leur fonction que l'on voudrait maintenant insister. 


\section{L'atteinte ressentie par les juges à leur fonction juridictionnelle}

Les juges rencontrés expriment leur embarras devant ce qu'ils ressentent comme une contradiction intrinsèque à l'objet de leur mission : décider dans les affaires qui leur sont présentées dans le respect, à la fois, des lois et de l'ordre public français et de l'intérêt concret de l'enfant considéré que la CEDH et la Cour de cassation les invitent désormais à prendre en compte. C'est ce qui se découvre dans l'extrait d'entretien suivant.

TGI 8, juge du siège, banlieue, 5 novembre 2015 :

C'est compliqué aussi cette notion de fraude, la Cour de cassation dans les arrêts Mennesson et la CEDH nous disent : si on pousse le raisonnement, il y a peut-être une fraude mais la fraude est de moindre importance par rapport à l'intérêt de l'enfant. [...] en fait on nous demande systématiquement de faire la balance entre l'intérêt de l'enfant et l'absence de fraude et le respect de l'ordre public et c'est vraiment l'injonction contradictoire en permanence ces dossierslà.

Les injonctions contradictoires entre lesquelles ils ont pris en étau finissent par conduire les juges à se demander si leur rôle est passé du maintien de l'ordre juridique à celui de chambre d'enregistrement. Ce malaise est ainsi décrit par un juge du siège du TGI 4 (12 novembre 2015) :

J'aimerais que ma position soit plus agréable, qu'on me dise ce qu'il en est exactement, parce qu'on dit ça sur les arrêts Mennesson sur la filiation mais ça laisse plein de points d'interrogation sur l'adoption et le respect de l'ordre international. [...] Qu'est-ce qu'on fait? C'est ce à quoi est confronté le procureur à Nantes, maintenant qu'on lui dit de transcrire la filiation issue d'une GPA, qu'est-ce qu'on fait? ça met mal à l'aise, on se dit est-ce qu'on a encore un rôle d'ordre ou pas, est-ce qu'on n'est qu'une chambre d'enregistrement?

Une même crainte sur la dévalorisation du rôle des magistrats se retrouve du côté du procureur de Nantes, responsable des transcriptions des actes de naissance établis à l'étranger. Il exprime clairement que prendre en compte l'intérêt de l'enfant serait abandonner sa mission de protection de l'ordre public (extrait du 13 novembre 2015) : 
La notion d'intérêt n'interfère pas dans mes décisions, elle ne doit pas parce que c'est un sujet, c'est une notion purement plastique qui est utilisée à toutes les sauces, qui a tendance à servir de paravent, de bouclier, de justificatif à n'importe quel procédé illégal ; c'est-à-dire qu'on vient mettre au pied du mur l'autorité française par un comportement qui est dès le départ vicié, c'est-à-dire que ces personnes-là savent pertinemment qu'elles violent la loi, [...] Par conséquent si je devais moi, introduire cette notion d'intérêt de l'enfant dans mon raisonnement juridique dès le départ ça veut dire que je mets totalement de côté l'ordre public, et ça a été la position de la Cour de cassation jusqu'à son arrêt du 3 juillet 2015, qui indiquait que l'intérêt de l'enfant ne devait pas supplanter la constatation de la violation de l'ordre public. [...] Voilà pourquoi pour moi je ne peux pas faire entrer en ligne de compte l'intérêt de l'enfant sous peine de quoi ça veut dire que j'abandonne totalement, je dirais, la référence à la loi.

L'intérêt de l'enfant, dès lors qu'il est interprété in concreto, est perçu comme l'instrument qui assure la prééminence du fait accompli sur le respect de la règle générale. Les juges craignent donc que le recours à l'intérêt de l'enfant entérine un véritable recul du droit, entraînant la démission du juge de son office : loin d'être encore la bouche de la loi, celui-ci ne serait que le porte-voix des pratiques individuelles.

\section{La GPA et la parenté d'intention}

Face à ces situations, les magistrats rencontrés, même si la décision de transcrire ou non l'acte de naissance établi à l'étranger n'est pas de leur ressort, se sont exprimés au sujet de l'établissement de la filiation du parent d'intention, notamment via l'adoption (par le conjoint ou la conjointe du père). Une série de questions, liées les unes aux autres, a émergé lors des entretiens. Notamment, comment faut-il interpréter l'exigence de la conformité à la réalité (article $47 \mathrm{C}$. civ:s) pour procéder à la transcription ? De quelle réalité s'agit-il ? Si l'état civil a été légalement dressé à l'étranger, ne peut-on considérer que sa transcription est

conforme à la réalité juridique constituée par l'acte de naissance régulièrement établi à l'étranger? Enfin, le refus de transcrire à l'état civil la parenté d'intention ne s'oppose-t-il pas à l'intérêt supérieur de l'enfant?

${ }^{36}$ Voir supra note 24. 
La plupart des magistrats rencontrés qui ont évoqué les décisions récentes (CEDH et Cour de cassation) les ont interprétées comme une injonction de transcrire la filiation paternelle parce qu'elle est conforme à la réalité biologique, du moins si on suppose que le père est le géniteur, ce dont la démonstration n'était pas en principe exigée. En revanche pour ces juges, les actes de naissance étrangers établissant parallèlement une parenté d'intention ne sauraient être retranscrits que partiellement, en y retranchant la mention du parent d'intention. Autrement dit, les juges interprètent la notion de réalité telle que mentionnée dans l'article 47 comme la réalité biologique, et non la réalité instituée par le jugement ou l'acte authentique étranger.

Le procureur de Nantes, responsable du service central d'état civil à Nantes s'exprime sans ambiguïté sur cette question, 13 novembre 2015 :

La Garde des Sceaux a publié une circulaire qui dans certains cas autorisait les greffiers en chef des tribunaux d'instance à délivrer des certificats de nationalité française à ces enfants lorsque les actes de naissance étrangers qui étaient produits par les parents d'intention étaient conformes à l'article $47 d u$ code civil, c'est-à-dire que grosso modo l'acte étranger était légalement établi dans le pays étranger et conforme aux lois étrangères et qu'il n'y avait pas d'irrégularités portant sur l'acte en question, que ce n'était pas un faux acte, où les faits qui étaient déclarés étaient exacts ; c'est bien sur ce dernier membre de phrase qu'il y a toujours aujourd'hui des questions qui sont non tranchées, c'est-à-dire que notamment lorsqu'on parle d'actes de naissance étrangers qui comportent dans leur contenu l'indication du père et de la mère, l'épouse n'ayant pas accouché, nous sommes sur une indication qui n'est pas conforme à la réalité puisqu'en droit de la filiation français est juridiquement considérée comme mère celle qui a accouché. Donc, l'épouse qui a demandé à une tierce personne, une autre femme, de porter son enfant pour son compte et qui est portée pour autant dans l'acte de naissance étranger, ça n'est pas conforme à la réalité.

Enquêtrice : la réalité là est toujours comprise comme la réalité biologique, ce n'est pas la réalité juridique du pays qui a établi l'acte?

\footnotetext{
${ }^{37}$ Il s'agissait de Madame Christiane Taubira à l'époque.
} 
Procureur : voilà, c'est une double réalité c'est-à-dire que nous considérons nous que ça ne correspond pas à la réalité biologique et, pas non plus à la réalité juridique française.

De surcroît le procureur de Nantes explique qu'il fait mener des recherches par les consulats pour connaître le droit local et vérifier que l'acte de naissance rédigé à l'étranger est bien conforme à ce droit :

On observe avec la pratique que, dans certains Etats américains notamment, on a dans des situations a priori semblables, mention de la mère porteuse ou mention de la mère d'intention ou aucune mention du tout. Donc, il y a a priori une application différente de la loi et moi il m'importe de savoir quel est ce droit local quand on le peut, donc là je fais faire des recherches par les consulats de France implantés dans ces Etats pour avoir le contenu de la loi étrangère. Donc si c'est effectivement permis dans le cas d'une GPA que le père souhaite ne pas faire apparaître la mère porteuse et que la loi locale autorise à établir un acte sans référence à la mère porteuse, je ne pourrais que transcrire, mais pour l'instant nous sommes en recherche des lois applicables pour savoir si cela est possible de transcrire. Il n'y a que pour l'Inde que la loi est tellement complexe, c'est une loi très ancienne qui date de l'époque ou l'Inde était encore sous domination britannique, ces textes étant tellement anciens on ne peut pas en tirer des enseignements, donc du coup vue l'ambiguïté de ces lois-là j'ai préféré faire profiter le doute aux personnes et je transcris dans ces cas-là. Quand j'ai un acte indien sans mention de la mère et que par ailleurs il est conforme au 47 qui vient le légaliser, j'autorise la transcription.

On voit donc comment la transcription de l'acte de naissance étranger est en réalité subordonnée à une triple exigence : il faut que l'acte soit conforme aux règles du pays où il a été dressé, aussi bien celles qui gouvernent la régularité formelle de l'acte, que celles qui gouvernent l'établissement de la filiation, conformément aux règles de droit international privé, mais il doit encore correspondre à la vérité biologique à laquelle serait assimilée « la réalité » mentionnée dans l'article 47 du Code civil français. Cette interprétation peut surprendre dans la mesure où le texte de loi français ne fait pas référence au lien biologique. Il faut souligner d'abord qu'il n'existe en droit français aucun cadre légal commun pour contrôler la validité des actes d'état civil étrangers. Les conditions de régularité, d'absence de falsification et de conformité à la réalité, posées par l'article 47 du Code civil, ne concernent 
que la force probante de l'acte d'état civil étranger, en tant qu'instrument de preuve du statut familial des personnes. Il semble abusif de se servir de cet article pour mettre à l'épreuve la validité intrinsèque de l'acte étranger au regard des règles françaises, en considérant que la réalité renvoie non pas à la réalité juridique constatée et établie dans l'acte de naissance étranger, mais à la réalité biologique supposée être au fondement du droit français de la filiation. La Cour de cassation s'est bien gardée en juillet 2015 de se prononcer sur le sujet puisque, on l'a dit, dans les espèces qui lui étaient soumises la réalité juridique et la réalité biologique (tout au moins physiologique) se confondaient. L'exigence d'un tel contrôle au fond, même sans prescrire une expertise génétique, des actes de naissance est-il légitime dans la mesure où il affecte l'identité des personnes et devrait à ce titre relever d'une action relative à la filiation ? Ne devrait-il pas dès lors être de la seule compétence du tribunal de grande instance (article 318-1 C. civ), et ne pas pouvoir être mis en œuvre à la seule initiative des autorités françaises en charge de la circulation internationale des personnes?

\section{Une convention internationale...et une révision de la loi ?}

Un dernier sujet a été abordé dans les entretiens, celui de la possibilité d'une convention internationale du type de celle de la Convention de la Haye sur la protection des enfants et la coopération en matière d'adoption internationale. Les positions sont loin d'être unanimes et reflètent celles entendues dans le débat social. Certains s'y montrent tout à fait opposés et plébiscitent à l'inverse des conventions bilatérales avec les différents pays autorisant la GPA pour qu'ils en refusent l'accès aux Français ; d'autres voient au contraire dans un accord international la seule solution pour encadrer une pratique irrépressible et réduire le risque d'exploitation des femmes les plus pauvres. Les deux extraits d'entretien suivants rendent compte de ces positionnements inverses :

TGI $n^{\circ} 1$ (très grande ville), juge du siège, 29 octobre 2015 ? :

Le seul moyen à mon sens de vraiment interdire ça c'est que la France passe des conventions avec les pays qui autorisent, par lesquelles ces pays s'engagent à ne pas autoriser la pratique à des ressortissants français ou de pays qui prohibent la pratique et qui sont du coup censé ne pas reconnaître les situations. Après dès lors que l'enfant existe c'est compliqué de...

TGI $n^{\circ} 7$ (grande ville), juge du parquet, vice-procureur, 16 décembre 2015 : 
Déjà je suis assez convaincue qu'il faut aller à l'international si on veut résoudre intelligemment le problème, car ça ne sert à rien de rester dans notre coin et qu'il y a une possibilité à l'étranger, on en arrive à une situation où ce sont les enfants qui payent. [...] Je pense que telle que la société est actuellement conçue où, quoiqu'on en pense, l'enfant est devenu un bien précieux, où on est prêt à tout pour concevoir un enfant, je pense plus à une réglementation intelligemment faite qu'à une prohibition qui sera contournée. Alors après la difficulté c'est de trouver un accord entre pays, parce que c'est vrai que là ce qui est problématique c'est que c'est devenu une source de profit dans certains pays étrangers au détriment des femmes qui se retrouvent exploitées ; tel que ça se conçoit dans certains pays ça s'apparente à une nouvelle forme d'esclavage moderne ce qui me gêne prodigieusement, donc nettement je suis pour la seconde solution qui permettrait d'encadrer la GPA en prenant en compte toutes les difficultés que pose cette question. "

Certains juges vont alors encore plus loin et considèrent que le législateur national n'a plus d'autre issue que de changer les textes qui mettent aujourd'hui les juges en difficulté.

TGI $n^{\circ} 1$, magistrat du siège, 29 octobre 2015 :

Moi je pense que ça va conduire le législateur à reprendre les article 16...ceux qui disent que c'est contraire à l'ordre public [...] ça reste quand même issu d'un mode de procréation qui est dit contraire à notre ordre public...donc estce qu'on doit faire complétement fi du code civil ? Si notre code civil est contraire à la convention européenne des droits de l'homme, [c'est] à notre législateur d'en tirer les conséquences et de réécrire, peut-être en n'autorisant pas de manière totalement libre, mais en rendant notre droit interne conforme à la convention européenne.

\section{Conclusions}

Malgré la condamnation de la France par la CEDH, la situation juridique des enfants nés à l'étranger du recours à une GPA reste problématique. Dans certains cas, l'enfant pourra avoir une filiation reconnaissant ses parents d'intention, notamment lorsque la filiation de la mère d'intention ou du deuxième père a été établie à l'étranger via une adoption. Mais dans la plupart des cas, seule la filiation paternelle sera établie laissant l'enfant privé de son autre parent d'intention, lorsque par exemple l'acte de naissance étranger de l'enfant mentionne 
deux pères ou bien une mère d'intention qui n'a pas accouché de l'enfant, du fait de l'interprétation biologique prédominante de l'article 47. Tel est le compromis auquel, de l'avis autorisé du Parquet de Nantes, aurait abouti la Cour de cassation le 3 juillet 2015.

On retiendra que cette solution contrainte par les récentes décisions de la $\mathrm{CEDH}$ et de la Cour de cassation place certains juges en conflit avec leur conception de leur identité professionnelle. Ainsi plusieurs parmi ceux interrogés ont exprimé un malaise devant ce qu'ils estiment être une contradiction dans les missions qui leur sont attribuées. Ils doivent décider en protégeant à la fois l'ordre public et l'intérêt de l'enfant. Selon eux, le respect de la loi commande de ne pas établir la filiation de l'enfant lorsque « la fraude à la loi » a présidé à sa naissance. Or, de l'avis de plusieurs juges, priver un enfant des parents qui ont souhaité sa venue au monde bafoue son intérêt. Plusieurs juges ont comparé ce dilemme à celui du prononcé de l'adoption internationale lorsque les conditions requises par la Convention de la Haye ne seraient pas satisfaites. Dans ce cas, comme dans celui de la GPA, le respect des principes légaux porte atteinte à l'intérêt, concrètement appréhendé, de l'enfant. Cette comparaison n'en est pas moins surprenante : comment mettre sur un même plan une loi nationale qui condamne purement et simplement une pratique de circulation d'enfant, la GPA, et une norme de nature internationale qui vise au contraire à encadrer cette autre forme de circulation d'enfants qu'est l'adoption, de façon à en prévenir les dérives ? La nature de ces textes est très différente et leur finalité paraît bien être contraire. La Convention de la Haye n'a pas pour objectif d'interdire l'adoption, mais de la réglementer de la manière la plus éthique possible. L'analogie entre l'ordre public français qui prohibe la GPA et le texte international témoigne d'une confusion qui en dit long sur le désarroi dans lequel les injonctions contradictoires de la loi nationale et des prescriptions européennes placent les juges français. Cette référence décalée à l'autorité de la convention de la Haye est sans doute une façon d'exprimer la dépossession de leur fonction juridictionnelle que ressentent certains juges lorsqu'ils se retrouvent contraints de mettre à l'écart l'ordre public français.

Quoi qu'il en soit, à invoquer le droit international privé, c'est bien plutôt l'appel à l'élaboration d'une convention sur le modèle de celle sur l'adoption, pour encadrer les pratiques transfrontalières de GPA et protéger les personnes les plus vulnérables, qui apparaîtrait logique. Quelques juges n'ont d'ailleurs manqué de souligner que ce serait là la seule façon de "résoudre intelligemment le problème "! Mais sur cette piste, déjà ouverte (voir les travaux de la Conférence de La Haye de droit international privé en 2014 et 2016), 
la route sera longue...Dans l'attente ou parallèlement, un encadrement de la GPA en droit français ne devrait-il pas être envisagé ? Au moins deux juges l'ont suggéré. Certes la Cour européenne a pris soin de reconnaître à chaque Etat le droit d'interdire le recours à la GPA sur son territoire; elle a seulement condamné les obstacles posés à la reconnaissance de la filiation des enfants nés des pratiques de gestation pour autrui réalisées à l'étranger. Sécuriser la filiation des enfants suffit pour mettre le droit français en conformité avec les exigences européennes, nul besoin de renverser la prohibition des conventions de gestation pour autrui. Et pourtant comment l'éviter ? En effet, outre que la solution d'un compromis a minima favorise les familles les plus fortunées qui peuvent seules assumer le coût important d'une gestation pour autrui délocalisée, elle place les juges face à des missions contradictoires : sanctionner les pratiques de gestation pour autrui quand elles ont lieu sur le territoire national et les accueillir lorsqu'elles sont de nature internationale conduit à leurs yeux à une dénaturation de leur fonction, comme on l'a montré. Il n'est donc pas étonnant que certains juges aillent jusqu'à préférer que l'interdiction de la gestation pour autrui soit révisée plutôt que de laisser leurs valeurs professionnelles ainsi atteintes.

\section{Bibliographie}

Académie nationale de médecine (2014). La Gestation pour autrui au regard du mariage entre personnes de même sexe, http://www.academie-medecine.fr/wpcontent/uploads/2014/05/27.5.14-HENRION-info-site.pdf.

Agacinski, S. (2009). Corps en miettes, Paris, Flammarion, 100 pp.

Almeling, R. (2011). Sex cells: the medical market for eggs and sperm, Berkeley and Los Angeles, California, University of California Press, 230 pp.

Badinter, E. (1980). L'amour en plus : Histoire de l'amour maternel (XVIIe-XXe), Paris, Flammarion, 372 pp.

Badinter, E., I. Théry, G. Delaisi, M. Godelier, M. Gross, S. et D. Menneson, I. Nisand, S. Portelli, H. Lancelin et A. Urwicz (2012), Mariage pour tous : la gestation pour autrui ne doit pas être le bouc émissaire, Le Monde, 19 décembre 2012, http://www.lemonde.fr/idees/article/2012/12/19/mariage-pour-tous-la-gestation-pour-autruine-doit-pas-etre-le-bouc-emissaire_1808271_3232.html.

Baudouin, J-L. et C. Labrusse-Riou (1987), Produire l'homme de quel droit? Etude juridique et éthique des procréations artificielles, Paris, Presses Universitaires de France, 288 p. 
Borrillo, D. (2016). La GPA, une liberté fondamentale, Libération, 22 décembre 2016, http://www.liberation.fr/debats/2016/12/22/la-gpa-une-liberte-fondamentale_1536967

Brunet, L. (2013) (dir.). A comparative study on the regime of surrogacy in EU Member States, Directorate General for Internal Policies, Legal and Parliamentary Affairs, May 2013, 378 p.

Brunet, L. (2015). Les atermoiements du droit français dans la reconnaissance des familles formées par des couples de femmes, Enfances Familles Générations, n²3, p. 91-89.

Bühler, N. (2016). Faire la parenté. Naturalisation stratégique et division de la maternité dans le recours transfrontalier au don d'ovocytes, Présentation au colloque international «Gestation pour autrui: dialogue entre le droit et les sciences sociales», Lausanne, Suisse.

Bureau, M.-F., et É. Guilhermont. (2011) Maternité, gestation et liberté : réflexions sur la prohibition de la gestation pour autrui en droit québécois, Revue Droit \& Santé McGill, vol. $4, \mathrm{n}^{\circ} 2$, p. $45-76$.

Busnel, M-C., R. Frydman, M. Szejer et J.-P. Winter, J-P. (2010). Abandon sur ordonnance. Manifeste contre la légalisation des mères porteuses, Montrouge, Bayard, 86 p.

Cadiet L. et E. Jeuland (2016). Droit judiciaire privé, LexisNexis, 950 p.

Chenedé, F. (2014). Les arrêts Mennesson et Labassée ou l'instrumentalisation des droits de l'homme, Dalloz, p. 1797.

Conférence de La Haye de droit international privé.

- (2014). Etude sur la filiation juridique et questions découlant des conventions de maternité de substitution à caractère international, Doc. prél. No $3 \mathrm{C}$.

- (2014). Opportunité et possibilité de poursuivre les travaux menés dans le cadre du projet filiation/maternité de substitution, Doc. prél. No 3 B.

- (2016). Note d'information pour la réunion du groupe d'experts relatif au projet Filiation/Maternité de substitution.

- (2016) Rapport de la réunion de février 2016 du du groupe d'experts relatif au projet Filiation/Maternité de substitution, Doc. prél. No 3.

Conseil d'Etat (2009). La révision des lois de bioéthique, Paris, La documentation française. http:/www.conseil-etat.fr/content/download/1177/3559/version/1/file/etudebioethique_ok.pdf.

Catto, M-X. (2013). La gestation pour autrui : d'un problème d'ordre public au conflit d'intérêts, La revue des droits de l'homme,3, https://revdh.revues.org/201\#tocto3n1. 
Fabre-Magnan, M. (2013a). La gestation pour autrui, fictions et réalité, Paris, Fayard, $128 \mathrm{p}$.

Fabre-Magnan, M. (2013b). Le refus de la transcription, Dalloz, p. 2384-2386.

Frison-Roche, M. (2014). L'impossibilité de réguler l'illicite : la convention de maternité de substitution, Dalloz, p. 2184-2187.

Depadt-Sebag, V. (2004). De la nécessité d'une réforme de l'article 16-7 du Code civil relatif à l'interdiction de gestation pour autrui, Revue générale de droit médical, p. 135-155.

Fouque, A. (2009). Les enjeux de la gestation pour autrui, Le Débat, n¹57 (nov-déc 2009), p. 145-157.

Fulchiron, H. (2014). La lutte contre le tourisme procréatif: vers un instrument de coopération internationale?, Journal du droit international, avril-mai-juin 2014, $\mathrm{n}^{\circ} 2$, 563588.

Giroux, M. (2011). Le recours à l'adoption pour établir la filiation de l'enfant né d'une mère porteuse : entre ordre public contractuel et intérêt de l'enfant, Revue du Barreau, vol. 70 , p. $509-544$.

Gobert, M. (1992), Réflexions sur les sources du droit et les "principes d'indisponibilité du corps humain et de l'état des personnes (A propos de la maternité de substitution), Revue trimestrielle de droit civil, vol. 91, n³, p. 489-528.

Golombok, S., F. Maccallum, C. Murray, E. Lycett et V. Jadva (2006). Surrogacy families: parental functioning, parent-child relationships and children's psychological development at age 2 (English), Journal of child psychology and psychiatry and allied disciplines (Print), vol. 47, n², p. 213-222.

Gross, M. (2014). «Dénouer la filiation de la procréation» dans Neirinck C. et M. Gross, Parents-enfants : vers une nouvelle filiation?, Paris, La documentation française, p. 115-190.

Iacub, M. (2004). L'empire du ventre. Pour une autre histoire de la maternité. Paris, Fayard, 365 p.

Hammons, S. A. (2008). Assisted Reproductive Technologies: Changing Conceptions of Motherhood? Affilia, Journal of Women and Social Work, vol. 23, n³, 270-280.

Henrion, R. et C. Bergoignan-Esper, C. (2009). La gestation pour autrui: Rapport 0905, Bulletin de L'Académie nationale de médecine, vol. 193, n³, p. 583-618. 
Langevin, L. (2015). Maternité de substitution: exploitation ou négociation?, Présentation au 7e Congrès international des recherches féministes dans la francophonie, Université du Québec à Montréal, Québec.

Langevin, L. (2015). La Cour d'appel du Québec et la maternité de substitution dans la décision Adoption - 1445 ; quelques lumières sur les zones d'ombres et les conséquences d'une solution la moins insatisfaisante, Revue Juridique Thémis, vol. 49, n², 451-486.

Malaket, A. 2015. Maternité de substitution : quelle filiation pour l'enfant à naître ?, , Revue du Notariat, vol. 117 n², p. 229-243.

Marais, A. (2015). Résister au droit transgressif de la maternité pour autrui ? in Marais A., La procréation pour tous, Dalloz, p. 121-142.

Markens, S. (2007). Surrogate Motherhood and the Politics of Reproduction, Berkeley and Los Angeles, California, University of California Press, 277 p.

Mattei, J-F. (2011). «Discussion pour la gestation pour autrui (GPA », dans G. David, R. Henrion, P. Jouannet et C. Bergoignan-Esper (dir.), La gestation pour autrui, Académie nationale de médecine, Paris, Lavoisier, p. 71-90

Merchant, J. (2013). «AMP et gestation pour autrui aux Etats-Unis, éléments d'analyse » dans I. Théry (dir.), Mariage de même sexe et filiation, Paris, Editions de l'EHESS, p. 129-142.

Mirkovic, A. (2013). Précisions de la Cour de cassation quant aux conséquences du recours à la GPA à l'étranger, La Semaine Juridique, Edition Générale, vol. 39, p. 985.

Moore, B. «Maternité de substitution et filiation en droit québécois », dans Liber amicorum. Mélanges en l'honneur de Camille Jauffret-Spinosi, Paris, Dalloz, 2013, p. 859874.

Neirinck, C. (2015). «La filiation, une question juridique » dans Neirinck, C. et M. Gross, Parents-enfants : vers une nouvelle filiation? Paris, La documentation française, p. $15-112$.

Nisand, I. (2010). Grossesse pour autrui: pour le cas par cas, Gynécologie Obstétrique \& Fertilité, vol. 38, n 3, p. 226-229.

OPECST (2008). La loi bioéthique de demain. Rapport de MM. Alain Claeys et JeanSébastien Vialatte, http://www.senat.fr/rap/r08-107-1/r08-107-1-syn.pdf.

Savard, A.-M. (2015). «L'établissement de la filiation à la suite d'une gestation pour autrui : le recours à l'adoption par consentement spécial en droit québécois constitue-t-il le moyen le plus approprié ? », dans C. LANDHEER-CIESLAK et L. LANGEVIN (dir.), La 
personne humaine, entre autonomie et vulnérabilité. Mélanges en l'honneur d'Édith Deleury, Montréal, Éditions Yvon Blais, p. 589-620.

Sénat (2008). Contribution à la réflexion sur la maternité pour autrui, Rapport d'information n421 (2007-2008), https://www.senat.fr/rap/r07-421/r07-421.html.

Tabet, P. (1998). La construction sociale de l'inégalité des sexes : des outils et des corps. Paris, L’Harmattan « Bibliothèque du féminisme », $208 \mathrm{p}$.

Terré, F et D. Fenouillet (2012). Droit civil, Les personnes. Paris, Dalloz, 934 p.

Trimming, K. et P. Beaumont (2013). International surrogacy arrangements: legal regulation at the international level, Hart Publishing, 559 p. 